\section{UNIVERSAL MACHINE FOR PROTOTYPE PRODUCTION}

ANTONIN DURNA, JIRI FRIES

VSB - Technical University of Ostrava, Faculty of Mechanical Engineering Ostrava, Czech Republic

DOI : 10.17973/MMSJ.2020_03_2019151

e-mail : antonin.durna@vsb.cz iiri.fries@vsb.cz

With development of new technologies the production is growing as well. Considering this the emphasis is placed on effectiveness and speed with which it is possible to test and make a new product. A 3D printer is an ideal utility in the area of prototyping. It is applicable for fast production of covers, ergonomic component and also small function elements. It is often necessary to design simple electronics in various areas of industry and at the same time to be able to test it. In this article we elaborated a possibility of replacement of a working head of a 3D printer with parallel kinematic in exchange for a head for subtle engraving of predominantly printed circuit boards. The main body of the 3D printer's effector is equipped with exchangeable system. It is enough to disconnect control cables, undo arrestment and replace working desk. Then it is necessary to connect controlling electronic. Moreover it is essential to improve working table. The working desk with clamping system is placed on the heated bed printer.

KEYWORDS

3D printer, engraving, printed circuit board, spindle

\section{INTRODUCTION}

The possibility of connecting a 3D printer with an engraving machine is very convenient for the quick and easily creation of a prototype. Let us have a head made up of a nozzle assembly, and a cooling unit for 3D printing. Furthermore, we need a head that is capable of milling printed circuit boards.

Since the milling of circuit boards means taking very small layers of tenths of millimetres away, we can apply a spindle of compact dimensions, using rather the recommended values for such an instrument than using a spindle directly designed for such milling. Another reason here is that the 3D printer structure with its parallel kinematics cannot bear an engraving head of large masses and dimensions, or unnecessarily limit possibilities in this structure.

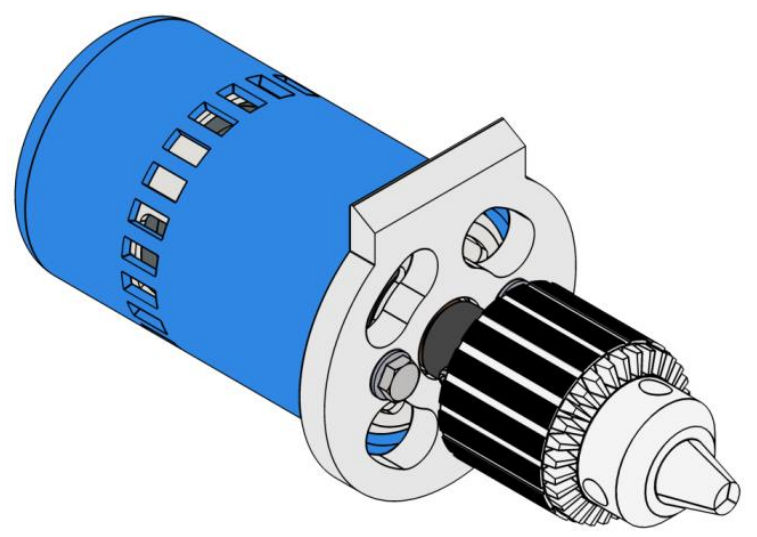

Fig. 1 A head assembly for engraving

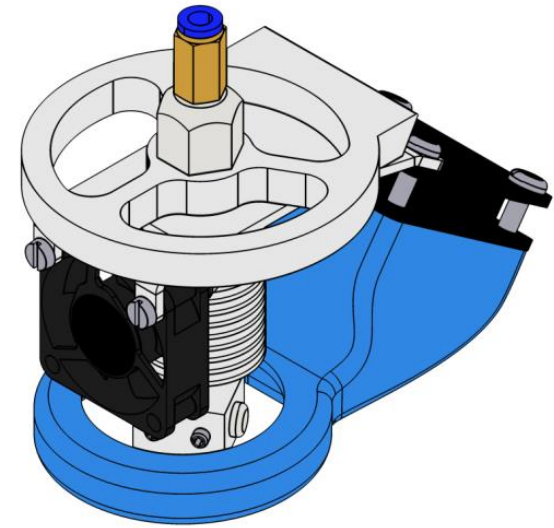

Fig. 2 A head assembly for printing

The head is made up of a clamping plate, which is of a circular shape, equipped with a nose with a chamfer. Each clamping plate is adapted to its specific modification of a work head. In Figure 1 and Figure 2 these modifications of work heads for 3D printing and engraving are illustrated.

The body of an effector is composed of a board (Fig. 3) made from aluminium alloy, with options to replace it and fix the work heads. The clamping mechanism is realized via an embedded circular pocket in the body of the effector. The pocket has a kerf with chamfered wall, which pushes the clamping plate against the face of the effector. When installing the clamping plate it is first inserted with the chamfered nose into the groove, and only then into the circular pocket. Then it is locked using a screw against loosening.

The body is assembled with arms, which must be in fixed the top and bottom using a flexible element (a spring, a rubber strip). In the case of articulated heads there is a tiny clearance which leaves visible marks on the printed work when the head moves. This simple but effective solution was used based of our practical knowledge.

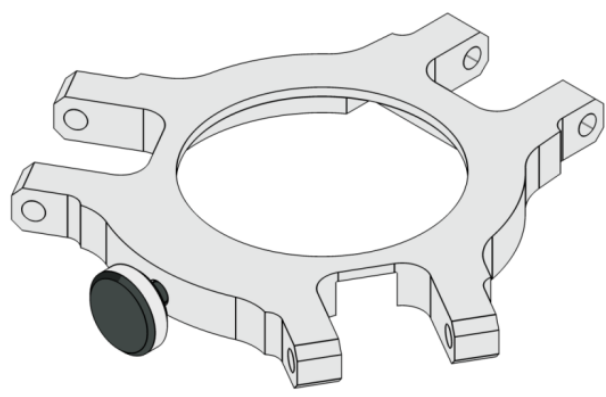

Fig. 3 A body of an effector

In addition to the effector adjustment it is also important to adjust the booms and the whole structure of the printer, because the engraving head is heavier than the head for 3D printing. Apart from the weight it is essential to take into consideration all conditions of printing and engraving, i.e. velocity and acceleration.

\section{KINEMATICS AND THE DISTRIBUTIONS OF FORCES ACTING ON THE WORK MECHANISM}

Figure 4 shows the working area of the printer in its plan view. Where $L_{N}$ is the length of the boom reflected in the plan view. $R_{F}$ is the radius of the effector from the boom axis, $x^{\prime}$ and $y^{\prime}$ are the distances from the beginning of the coordinate axes up to 


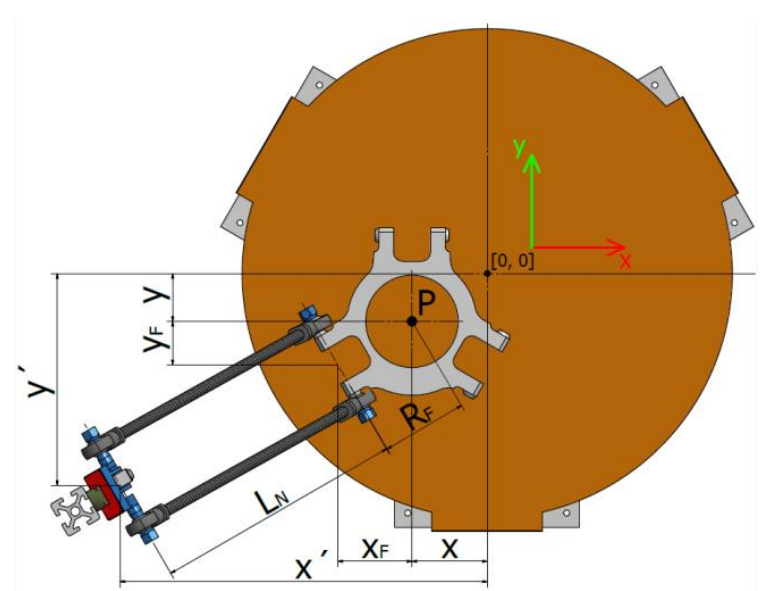

Fig. 4 Schematic illustration of the boom in the plan view

the guidance axis, which is given by the machine design. The $P$ point is placed in the centre of the effector, and it determines the position in the plane $x y$. The $x$ and $y$ values indicate the coordinates to move the $P$ point in the coordinate system, $x_{F}$ and $y_{F}$ is the distance from the axis of the effector to the centre of the booms.

The formula for the calculation of the unknown $L_{N}$ :

$$
\sqrt{\left(x^{2}+x-x_{F}\right)^{2}+\left(y^{2}+y-y_{F}\right)^{2}}=L_{\bar{M}}
$$

In Figure 5 there is a drawing of the printer, where $L_{R}$ is the length of the boom, $h_{p}$ the original height of a carriage for coordinates $[0,0,0]$, and it is given by the structure of a printer, $d_{p}$ indicates the truck trajectory after the $x$ and $y$ coordinates are adjusted. When we include drawings into these equations, and after a few adjustments are made, we obtain an equation expressing an unknown $d_{p}$ :

$\mathrm{d}_{p}=\sqrt{L_{i p}^{2}-\left(x^{2}+x-x_{p}\right)^{2}+\left(y^{2}+y-y_{p}\right)^{2}}-h_{p}$

To move the effector in the $z$ axis direction, all three booms must move to the same distance in the direction of movement. Figure 6 shows the general diagram of the forces acting on one boom. Where $F_{G T}$ is the weight of the boom with the articulated heads.

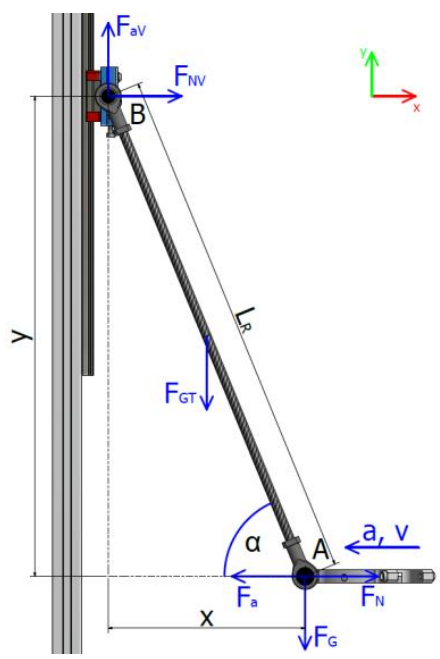

Fig. 6 The basic scheme

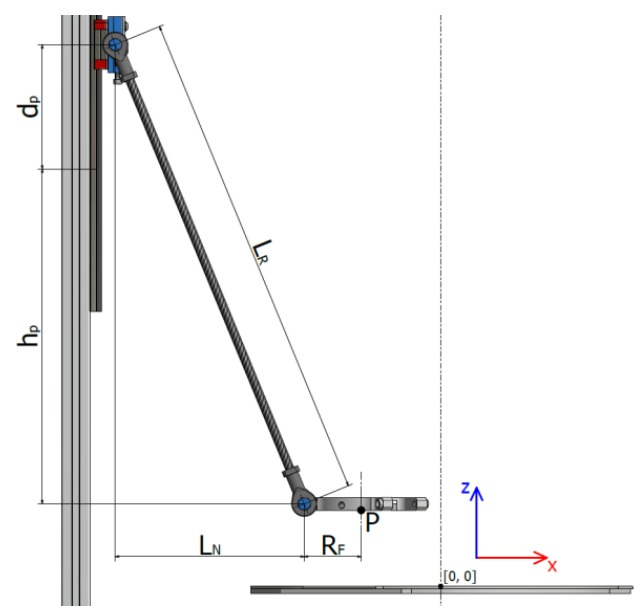

Fig. 5 Schematic of the boom in the drawing

From the basic equations for static balance the reactions acting on points $A$ and $B$ can be calculated.

Then we can determine the static motor torque. Where $r_{R}$ is the radius of the propulsion roller and $m_{V}$ is the weight of the entire carriage.

The equation for lifting the carriage [Marek 2010]:

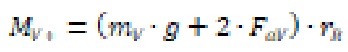

For lowering the carriage [Marek 2010]:

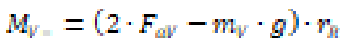

Subsequently derived equation for the calculation of the required static torque for the motor:

$M_{\mathrm{Bg}}=\frac{M_{\mathrm{Fc}}}{\eta_{\mathrm{LP}}}$

Where $\eta$ is the overall efficiency of the mechanism.

\subsection{The calculation of the propulsion}

Using the derived equations for static, kinematic and dynamic aspects we can obtain the necessary static and dynamic motor torque. In this example is $m_{V}=0.072 \mathrm{~kg}, m_{T}=0.230 \mathrm{~kg}, m_{G}=$ $0.474 \mathrm{~kg}, \mathrm{~m}_{\mathrm{GT}}=0.015 \mathrm{~kg}, r_{\mathrm{R}}=6.11 \mathrm{~mm}$. Where $m_{T}$ is the mass of the effector with a printing head, $m_{G}$ the weight of effector with a head for engraving, and $m_{G T}$ the weight of a boom.

Where for the weight of the effector $m$ we will initially use $m_{T}$ :

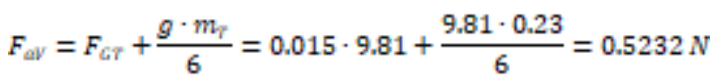

The unknown $F_{N V}$ and $F_{N}$ vary depending on the angle of tilt $\alpha$, are therefore the calculated values are plotted onto the graph. For the calculations we used the angle of tilt within the interval $\left[81.8^{\circ} ; 27.07^{\circ}\right]$, which is the angle at which the effector moves over the entire workbench [Durna 2017]. 


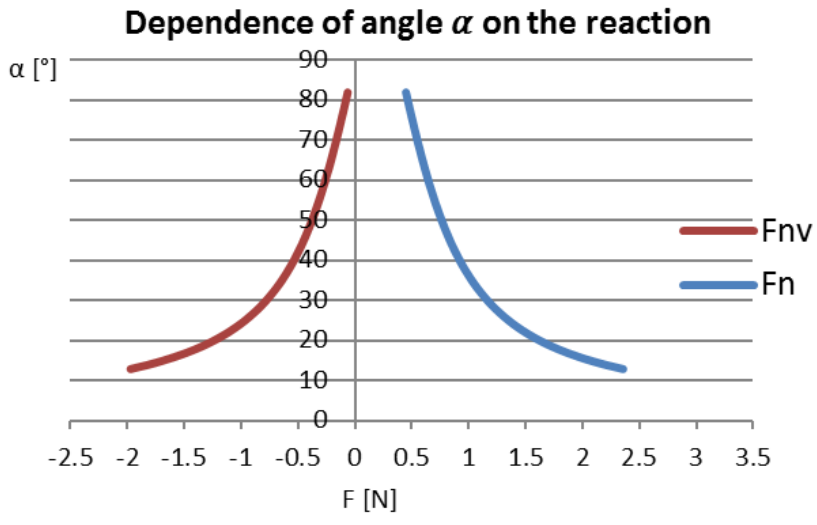

Fig. 7 Graph on the reaction course $F_{N V}$ and $F_{N}$

Transfer between the motor and the boom carriage is realized using a synchronous belt 2MGT3-6 of $1.850 \mathrm{~mm}$, and a belt pulley with the GT2 profile with 20 teeth 20-2GT-6.

Where the equation for the torque required to lift the carriage is:

$M_{\text {WD }}=(0.072-9.81+2 \cdot 0.5232)-0.00611=0.0107 \mathrm{Nm}$

As well as the equation for starting the carriage:

$M_{P ळ}=(2 \cdot 0.5232-0.072-9.8)-0.00611=0.00207 \mathrm{Nm}$

Then the equation for calculating the required static torque is:

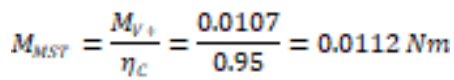

Where $\mathrm{n} \eta_{c}$ is the overall efficiency obtained:

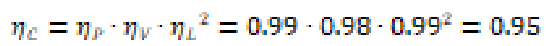

$\eta_{\mathrm{P}}=0.99$ is the efficiency of the belt drive, $\eta_{\mathrm{v}}=0.98$ rolling lines, $\eta_{L}=0.99$ bearings

The maximum specified print velocity $v=300 \mathrm{~mm}^{-} \mathrm{s}^{-1}$ and acceleration of effector $a=10000 \mathrm{~mm}^{-} \mathrm{s}^{-2}$. The values of the acceleration and velocity for the point $B$ were calculated from the derived equations, based on an analytical solution. This solution is thus based on analytical geometry, and on the application of fundamental equations for kinematics. Then a graph was plotted that shows the course of the acceleration, and the velocity of the point $B$ from Fig. 6 .

\section{Acceleration and velocity of point $B$}

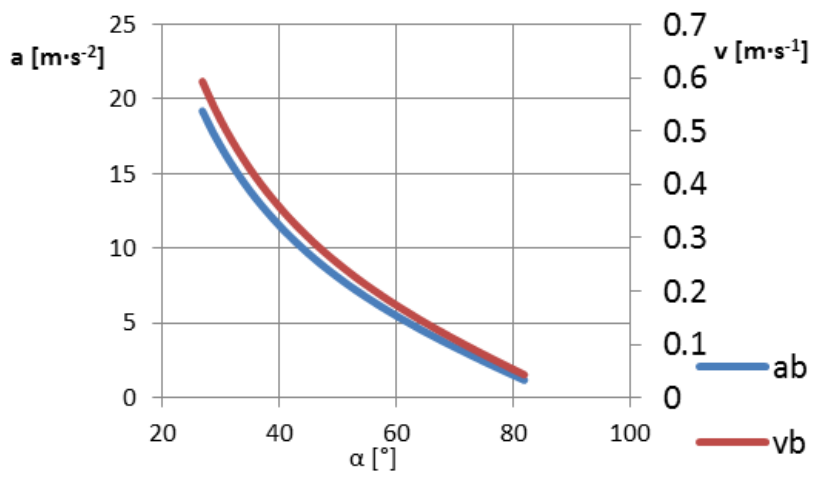

Fig. 8 The graph of the acceleration course and velocity of the boom carriage (B point)
Where the greatest velocity $\mathrm{v}_{\mathrm{Bmax}}$ is obtained:

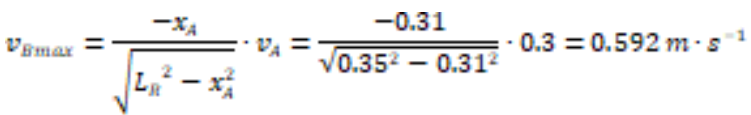

In equation 12 the negative sign $\left(-x_{A}\right)$ is very important, which gives unique information on the direction of movement. A positive sign indicates the direction of movement in the direction of increasing coordinates, a negative sign conversely in the direction of descending coordinates. This negative sign in the above written equation means that when the point $A$ travels to the right, the point $B$ will decline, and vice versa [VSBTUO 2011].

Then we can determine the engine velocity:

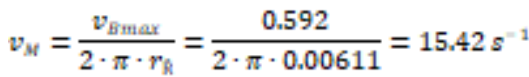

The largest acceleration $a_{B M A X}$ is determined:

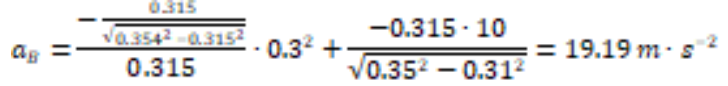

From the calculated largest acceleration of the point $B$ it is possible to specify the largest angular acceleration of the motor:

$\varepsilon_{\text {i⿱ }}=\frac{a_{E}}{\eta_{R}}=\frac{19.19}{0.00611}=3142.266 \mathrm{rad}-\varepsilon^{-2}$

Where the moments of inertia of the belt pulley and tensioner are: $\mathrm{J}_{\mathrm{R}}=0.207 \mathrm{~kg} \cdot \mathrm{mm}^{2}, \mathrm{~J}_{\mathrm{K}}=0.079 \mathrm{~kg} \cdot \mathrm{mm}^{2}$

$M_{\text {WDDP }}=\left(2.07 \cdot 10^{-98}+7.9 \cdot 10^{-9}\right)-3142.26+0.0107=0.011$

If we add into the same equations the weight of the effector equipped with the engraving head, we will get the values:

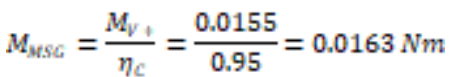

$M_{\text {wase }}=\left(2.07 \cdot 10^{-9}+7.9 \cdot 10^{-0}\right) \cdot 3142.26+0.0163=0.016$

\subsection{Propulsion design}

A stepper drive is used here for the drive. Stepper motors are suitable for applications which need accurate steering at low and medium velocities. Their advantages include their low price and the minimum requirements for maintenance. When applying stepper motors a torque reserve of $50 \%-100 \%$ is generally recommended for a sufficient torque margin at e.g. the impact loading of the torque moment [Marek 2010].

As the power supply to the motor, a DC voltage of $24 \mathrm{~V}$ is used. In comparison of the values calculated with the torque characteristics of Fig. 9 a Microcon SX17-1005LQCEF [Microcon 2018] stepper motor with a series connection is sufficient. All the basic values of the motor are given in Table 1.

\begin{tabular}{|c|c|c|c|c|c|}
\hline Type & $\begin{array}{l}\text { Static } \\
\text { moment }\end{array}$ & $\begin{array}{l}\text { Rated } \\
\text { current }\end{array}$ & Resistance & Weight & $\begin{array}{l}\text { Step } \\
\text { length }\end{array}$ \\
\hline & {$[\mathrm{Nm}]$} & {$[\mathrm{A}]$} & {$[\Omega]$} & [kg] & {$\left[{ }^{\circ}\right]$} \\
\hline $\begin{array}{c}\text { SX17- } \\
\text { 1005LQC } \\
\text { EF }\end{array}$ & 0.52 & 1 & 5.9 & 0.3 & 1.8 \\
\hline
\end{tabular}


Table. 1 Specification od stepper motor [Microcon 2018]

Torque characteristic of the stepper motor

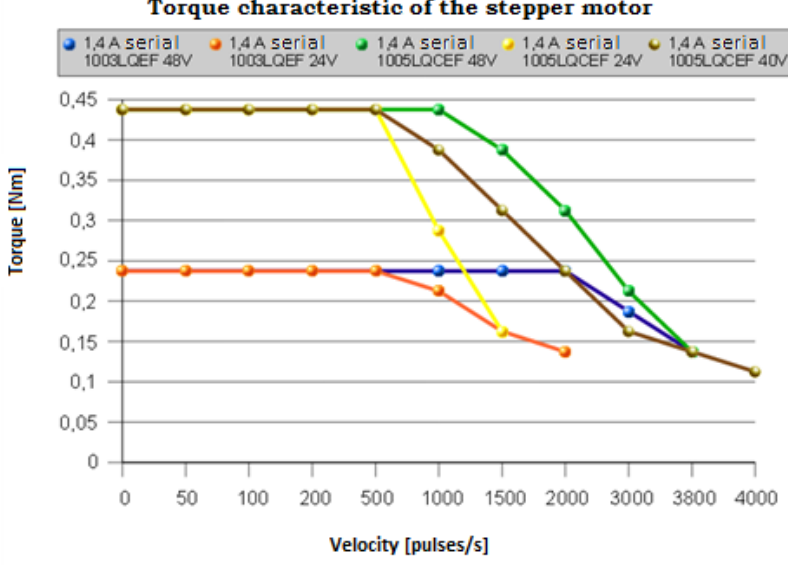

Fig. 9 Torque characteristic of the stepper motor [3]

For engine control AND4982 Allegro drivers are used, which allow $1 / 16$ micro-stepping, and thus can divide one step of this motor into another 16 . The length of one step is $1.8^{\circ}$, and one revolution of the engine makes 200 steps. The total number of steps needed to feed $1 \mathrm{~mm}$ on the $Z$ axis can be calculated:

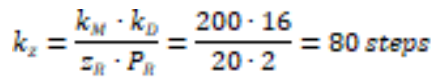

From this we can calculate the minimum distance for one step on the $Z$ axis:

$z_{\min }=\frac{1}{k_{2}}=\frac{1}{80}=0,0125 \mathrm{~mm}$

The feed of the $Z$ axis by one step of this motor:

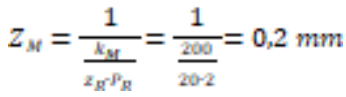

\section{DESIGN OF WORKBENCH}

A workbench is firmly attached to the frame, enabling the exchange of job boards for 3D printing and engraving. It consists of two fixed and one sliding jaw to fasten the boards. Each jaw is equipped with a FSR402 [FSR 402] pressure sensor to set the zero point of the $Z$ axis, which also works as a limit switch. Between the heating board for 3D printing and the pressure sensor there is placed a Teflon insulation pad. The upper edge of each jaw has a chamfered nose which presses the work boards. In Table 2 you can see the basic parameters of the pressure sensor.

\begin{tabular}{|l|c|c|}
\hline Type & {$[-]$} & FSR402 \\
\hline Range of detection & {$[\mathrm{g}]$} & $10-10000$ \\
\hline Thickness & {$[\mathrm{mm}]$} & 0.45 \\
\hline Temperature range & {$\left[{ }^{\circ} \mathrm{C}\right]$} & $-30-70$ \\
\hline Lifetime & {$[-]$} & 10 million switching \\
\hline
\end{tabular}

Tab. 2 specification of pressure sensor [FSR 402]

At the time the print nozzle travels above the work desk and touches the panel, the circuit closes and thus the zero point of the $\mathrm{Z}$ axis is set. When replacing the job boards there is no need to meet the same precise flatness of the working area. Thanks to the deployment of the pressure sensors on the three points of the plane, you can set the controlling firmware so that when the zero point is set it touches the work board in several places and thus the incline of the work board is identified. Then the working software can correct this inequality and the final product will not be affected.

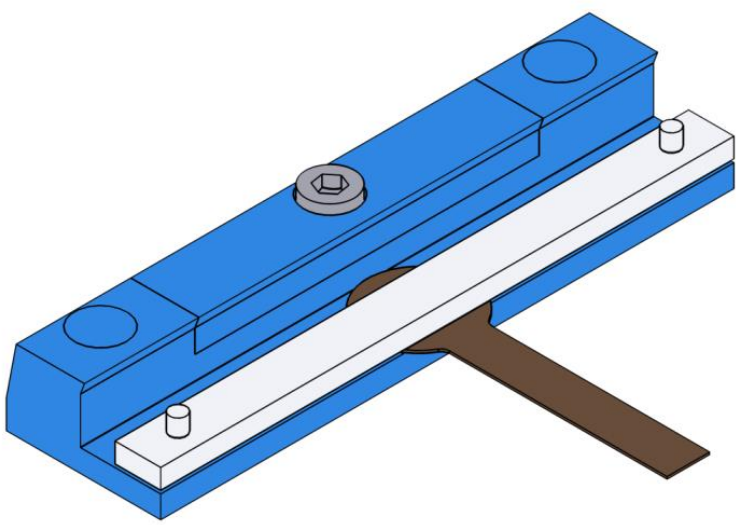

Fig. 10 The holder with load cell and insulation pad

\subsection{Design od head bed}

The work bed heating for the $3 \mathrm{D}$ printing is realized via resistive heating using a printed circuit. It is located on the $3 \mathrm{~mm}$ thick aluminium plate that is fixed on the sub-frame using jaws. The bed is powered by $24 \mathrm{~V}$ DC voltage. It can reach a maximum temperature of $180^{\circ}$. On the bottom of the board the NTC $100 \mathrm{~K}$ thermistor is located for temperature sensing and switching the heating process on.

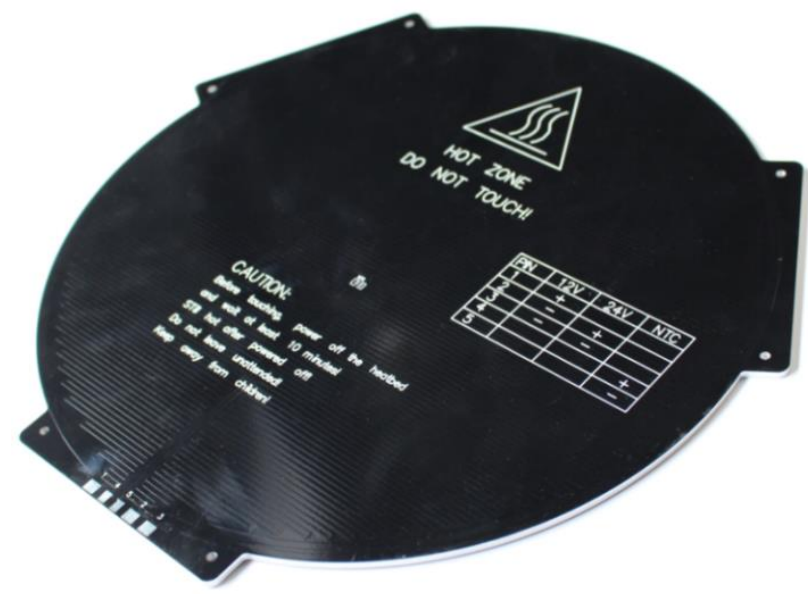

Fig. 11 Heat bed MK3 ALU Dual Power

Another pad is placed on the heating bed, made from a $2 \mathrm{~mm}$ thick aluminium plate or glass. On it there is applied a layer for printing.

\subsection{Engraving workbench}

The workbench for engraving is made of beechwood or plywood. In practice it was verified that plywood is sufficient and an inexpensive option here. It is fitted with a system of holes to mount clamps. These clamps are fixed using carriage bolts. Adjustment of the $\mathrm{Z}$ axis is provided using the contact of the engraving tool with the processed copper-plated board. Both the tool and the board are linked into the circuit, and when they touch the circuit is closed, and thus the zero coordinate is detected. The workbench is fixed in the same way as the board for the 3D printing, using jaws, and it is placed on a heated pad. 


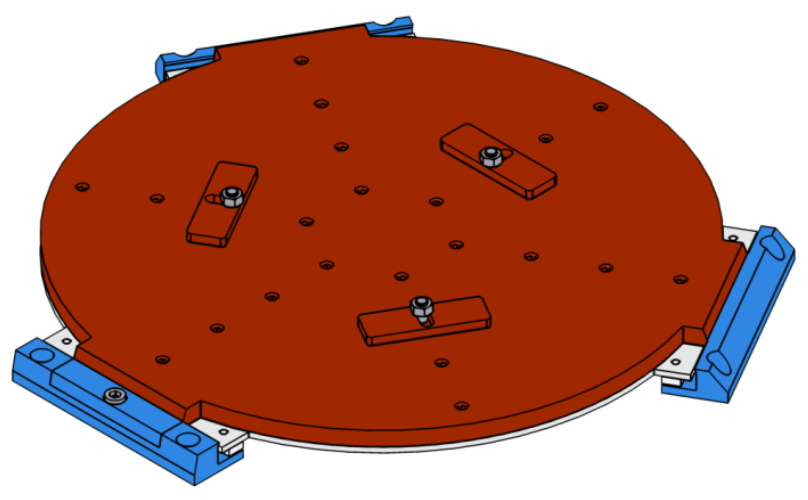

Fig. 12 The engraving bench with jaws

\section{CONCLUSIONS}

When designing such a machine tool, it is important to consider the weight of the effector, which can significantly limit the possibilities of the machine, even when it is used only for 3D printing. This is caused merely by the weight of the adjusted effector, which enables the heads to be replaced. The greatest weight will bear the head with an engraving spindle. However, for these operations it will not reach the same velocities as for $3 \mathrm{D}$ printing, and thus there is no increase in stress on the parts, and a more powerful drive is not needed here. For the drive design it is advisable to use calculations for graph compilation shown in Fig. 8, and it is advisable to start with the greatest acceleration and velocity for the $B$ point, which is modified by using a change of angle alpha. The workbench can be adjusted in more ways. The bench can be equipped with another clamping system, or we can replace the workbench for a heated board. Nevertheless, it is necessary to take into consideration the setting of the $\mathrm{Z}$ axis above the bench, and its planeness. One advantage here is the possibility of compensating the inclination of the work bench, using some Gcode generating software. A structure modified like this enables us to use one machine tool for two purposes, and thus increase the productivity in manufacturing prototypes, while lowering their costs.

\section{ACKNOWLEDGMENTS}

Article has been done in connection with project Research and innovations of modern technologies in manufacturing practice and Research and development of sophisticated technologies in industrial practice reg. no. SP2019/2 and SP2020/1 respectively supported by Specific Research Programme financed by The Ministry of Education, Youth and Sports.

\section{REFERENCES}

[Durna 2017] Durna, A., Fries, J. Modification of the nozzle assembly in a 3D printer for printing materials with higher melting temperatures. In SGEM 2017; 29 June-5 July, 2017, Albena, Bulgaria. Volume 17. Issue 62. Sofia : STEF92 Technology Ltd., 2017, 923930 pp. DOI: 10.5593/sgem2017/62/S28.118.

[FSR 402] Trossen Robotics. Downers Grove [online]. 2016 Available from http://www.trossenrobotics.com/productdocs/2010-10-26-DataSheet-FSR402Layout2.pdf

[Marek 2010] Marek J, A.A. and Blecha P, B.B. Designing of CNC Machine Tools . 2st ed. Praha: MM publishing, 2010. MM special. ISBN 978-80-254-7980-3.

[Microcon 2018] Microcon. Praha 9 [online]. 2016. Available from http://microcon.cz/.

[VSB-TUO 2011] VSB - Technical University of Ostrava Department of Applied Mechanics, Title of electronic journal [online]. 2011. Available from https://www.fs.vsb.cz/export/sites/fs/330/.content /files/Aplikovana_mechanika_03_prednaska.pdf.

\section{CONTACTS:}

Ing. Antonin Durna

VSB-Technical University of Ostrava,

Department of Production Machines and Design

17. listopadu $2172 / 15$

708 00, Ostrava - Poruba, Czech Republic

e-mail: antonin.durna@vsb.cz

Tel.: +420 597324271

www.340.vsb.cz

Assoc. prof. Ing. Jiri Fries, Ph.D

VSB-Technical University of Ostrava,

Department of Production Machines and Design

17. listopadu 2172/15

708 00, Ostrava - Poruba, Czech Republic

e-mail: jiri.fries@vsb.cz

Tel.: +420 597324207

www.340.vsb.cz 